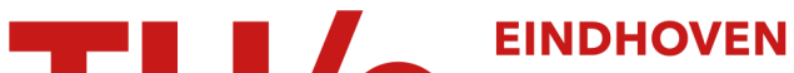 UNIVERSITY OF TECHNOLOGY
}

\section{Hydrogel/poly-dimethylsiloxane hybrid bioreactor facilitating 3D cell culturing}

Citation for published version (APA):

Schurink, B., \& Luttge, R. (2013). Hydrogel/poly-dimethylsiloxane hybrid bioreactor facilitating 3D cell culturing. Journal of Vacuum Science and Technology, B, 31(6), [06F903]. https://doi.org/10.1116/1.4831762

DOI:

10.1116/1.4831762

Document status and date:

Published: 01/01/2013

\section{Document Version:}

Publisher's PDF, also known as Version of Record (includes final page, issue and volume numbers)

\section{Please check the document version of this publication:}

- A submitted manuscript is the version of the article upon submission and before peer-review. There can be important differences between the submitted version and the official published version of record. People interested in the research are advised to contact the author for the final version of the publication, or visit the $\mathrm{DOI}$ to the publisher's website.

- The final author version and the galley proof are versions of the publication after peer review.

- The final published version features the final layout of the paper including the volume, issue and page numbers.

Link to publication

\section{General rights}

Copyright and moral rights for the publications made accessible in the public portal are retained by the authors and/or other copyright owners and it is a condition of accessing publications that users recognise and abide by the legal requirements associated with these rights.

- Users may download and print one copy of any publication from the public portal for the purpose of private study or research.

- You may not further distribute the material or use it for any profit-making activity or commercial gain

- You may freely distribute the URL identifying the publication in the public portal.

If the publication is distributed under the terms of Article $25 \mathrm{fa}$ of the Dutch Copyright Act, indicated by the "Taverne" license above, please follow below link for the End User Agreement:

www.tue.nl/taverne

Take down policy

If you believe that this document breaches copyright please contact us at:

openaccess@tue.nl

providing details and we will investigate your claim. 


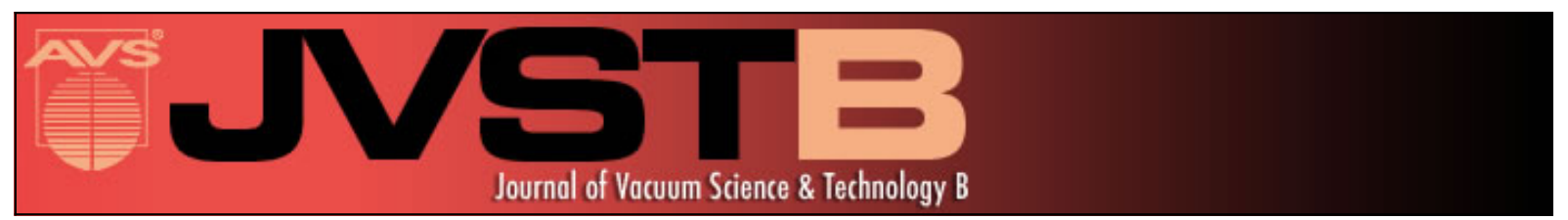

\section{Hydrogel/poly-dimethylsiloxane hybrid bioreactor facilitating 3D cell culturing}

Bart Schurink and Regina Luttge

Citation: Journal of Vacuum Science \& Technology B 31, 06F903 (2013); doi: 10.1116/1.4831762

View online: http://dx.doi.org/10.1116/1.4831762

View Table of Contents: http://scitation.aip.org/content/avs/journal/jvstb/31/6?ver=pdfcov

Published by the AVS: Science \& Technology of Materials, Interfaces, and Processing

\section{Articles you may be interested in}

Dynamic 3D cell culture via a chemoselective photoactuated ligand

Biointerphases 9, 031005 (2014); 10.1116/1.4881035

Comparative assessment of the stability of nonfouling poly(2-methyl-2-oxazoline) and poly(ethylene glycol) surface films: An in vitro cell culture study

Biointerphases 9, 031003 (2014); 10.1116/1.4878461

Flow bioreactor design for quantitative measurements over endothelial cells using micro-particle image velocimetry

Rev. Sci. Instrum. 84, 045109 (2013); 10.1063/1.4802681

Covalently immobilized biomolecule gradient on hydrogel surface using a gradient generating microfluidic device for a quantitative mesenchymal stem cell study

Biomicrofluidics 6, 024111 (2012); 10.1063/1.4704522

Non-positional cell microarray prepared by shape-coded polymeric microboards: A new microarray format for multiplex and high throughput cell-based assays

Biomicrofluidics 5, 032001 (2011); 10.1063/1.3608130

\section{iOnLINE}

Select Si Ge Au and more for Advanced Nanofabrication www.raith.com
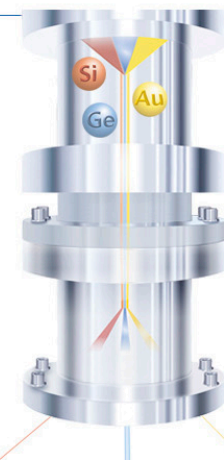


\title{
Hydrogel/poly-dimethylsiloxane hybrid bioreactor facilitating 3D cell culturing
}

\author{
Bart Schurink $^{\text {a) }}$ \\ Mesoscale Chemical Systems, MESA+ Institute for Nanotechnology, University of Twente, P.O. Box 217, \\ 7500 AE Enschede, The Netherlands \\ Regina Luttge \\ Mesoscale Chemical Systems, MESA+ Institute for Nanotechnology, University of Twente, P.O. Box 217, \\ 7500 AE Enschede, The Netherlands and Department of Mechanical Engineering, Microsystems Group, \\ Eindhoven University of Technology, Den Dolech 2, 5600 MB Eindhoven, The Netherlands
}

(Received 21 June 2013; accepted 1 November 2013; published 15 November 2013)

\begin{abstract}
The authors present a hydrogel/poly-dimethylsiloxane (PDMS) hybrid bioreactor. The bioreactor enables a low shear stress 3D culture by integrating a hydrogel as a barrier into a PDMS casing. The use of PDMS allows the reversible adhesion of the device to a commercially available microelectrode array. A two-step molding process facilitates this relatively simple, cost effective, and leakage-free add-on microculture system. Agarose (2\%) is used as hydrogel barrier material and mass transport is evaluated by fluorescein isothiocyanate-albumin fluorescence under static conditions which yields a diffusion coefficient of average value of $2.2 \times 10^{-7} \mathrm{~cm}^{2} \mathrm{~s}^{-1}$ across the barrier. To validate our bioreactor for diffusion limited 3D cell culture, rat cortical cells were successfully cultured in Matrigel for 6 days. @ 2013 American Vacuum Society. [http://dx.doi.org/10.1116/1.4831762]
\end{abstract}

\section{INTRODUCTION}

For the past 20 years, there has been an increasing demand from the pharmaceutical industry for high throughput cell screening systems which can function as an in vitro model for the validation of drugs or toxicity screenings. ${ }^{1,2}$ In these systems, cells would be cultured in controlled environments for the study of cellular physiological and pathological responses.

To correlate such a model with in vivo conditions, the culturing of cells in a three-dimensional (3D) matrix has been proven to be of vital importance. ${ }^{3}$ Besides the difference in cell-cell and cell-extracellular matrix interaction, 3D systems outperform 2D cultures in their response to biochemical and biophysical factors. In general, 3D cultures better represent native cellular behavior in comparison to planar cultures. ${ }^{4,5}$ One way to establish $3 \mathrm{D}$ cultures is the use of a hydrogel matrix, consisting of cross-linked fibers with high water content that are abundantly found in native animal tissues. ${ }^{6}$ Taking these materials in vitro, the challenge for creating appropriate culture conditions arises from a lack of a vascular structure for the transport of oxygen and nutrients into the cell culture. In recent years, microfluidic culture systems, or so-called microbioreactors, have been developed to address this problem and enable the use of continuous flow and small culture volumes. ${ }^{7-9}$

Potentially, the research field of neurology can benefit from the fidelity of 3D culturing on a chip. ${ }^{1}$ Of particular interest are chips with planar microelectrode arrays (MEAs), which were developed for the study of in vitro neuronal networks and which are generally accepted tools in this field of research. The basic design of a MEA consists of a glass surface with embedded, densely packed microelectrodes for

$\overline{{ }^{a} \text { Electronic mail: B.Schurink@utwente.nl }}$ neuron coupling, which are connected to macropatch electrodes for the signal readout. ${ }^{10-12}$

One may think of a microfluidic bioreactor designed for 3D culturing on such a surface, while during culturing, the neurons remain coupled to an array of electrodes. Such advanced experimental tools may result in a more valid model for electrophysiology in combination with drug and toxicity screening.

Previously, polydimethylsiloxane (PDMS) has been used for the fabrication of microfluidic chips that can be sealed to a glass surface by spontaneous and reversible adhesion, ${ }^{13}$ which provides an appropriate technology platform for cell culturing. However, many of the microfluidic cells-on-a-chip designs exert high shear forces onto the cells and their matrices through the flow of culture medium. Therefore, these systems are limited in their utility for prolonged 3D culturing. ${ }^{14}$ An integrated barrier, separating the culture chamber from the culture medium channel, can avoid this direct shear stress onto the culture. Such a design sustains an appropriate mass transport and prevents the cells from migrating out of the culture into the channel.

Recently, microfluidic systems with an integrated hydrogel as a barrier for cell culture on a chip have been described. In these systems, cells are cultured inside of a straight channel layout (e.g., to create a stimuli gradient) in which a hydrogel barrier ensures a controlled inflow of nutrients and oxygen. ${ }^{15,16}$ Due to the fabrication by soft-lithography, that utilizes a photoresist mold of SU-8, the channels are restricted to a uniform height. This molding principle is limited in its maximal height to realize a vertically orientated 3D culture atop of the prearranged electrodes of a commercially available MEA. Although very thick and advanced multilayer SU-8 photoresist processing is described in literature, ${ }^{17}$ it is cumbersome to realize a mold structure by SU-8 with aligned parts at different heights. To cope with this limitation of an SU-8 mold, we introduce a fabrication process for a microfluidic bioreactor that complies 
to the dimensions of the MEA and provides a simple 3D addon microculture system. The fabrication of this microfluidic bioreactor is based on soft-lithography from a precision engineered metal mold.

Our bioreactor consists of a round culture chamber which is surrounded by a ring shaped channel separated by a hydrogel barrier. This design maximizes the surface coverage between the barrier and the culture to ensure a uniform inflow into the cell culture. By means of a two-step molding process using a polished mold surface with interchangeable parts, we ensure a leak-tight connection between the barrier, the PDMS casing and the MEA as well as a central alignment of the barrier, the flow channel and the culture within the PDMS casing.

In this paper, our main objective is the design of a novel hydrogel/PDMS hybrid bioreactor for reversible adhesion atop a commercially available MEA and the validation of the bioreactor for culturing 3D neuronal networks from dissociated primary brain cells.

\section{METHODS AND MATERIALS}

\section{A. Two-step molding process}

A two-step molding process is performed using an assembled mold (Fig. 1). Figure 1(a) shows all the interchangeable parts of the mold. The mold is based on a surface polished brass plate of $40 \times 40 \mathrm{~mm}$ (part A) and fabricated by computer numerical control milling (Mechanical workshop, University of Twente). The parts D and F are also

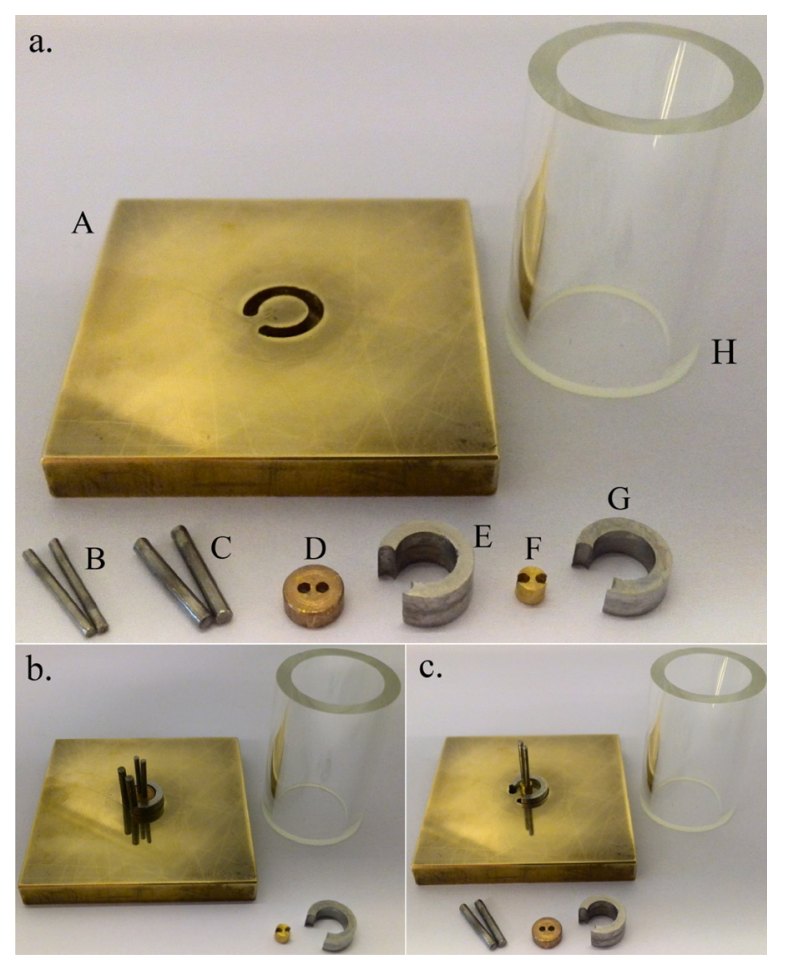

FIG. 1. (a) (Color online) Overview of the metal mold and its parts. (b) For the molding of the PDMS casing, parts B, C, D, and E are assembled into the mold baseplate (part A).The borosilicate glass tube (part $\mathrm{H}$ ) will be placed on the base plate during molding. (c) For the molding of the hydrogel barrier, parts B, G, and F are assembled into the mold base plate (part A). made of brass, whereas the other interchangeable parts $\mathrm{B}, \mathrm{C}$, $\mathrm{E}$, and $\mathrm{G}$ are made of stainless steel. The inner and outer diameters of parts $\mathrm{E}$ and $\mathrm{G}$ are 3 and $5 \mathrm{~mm}$, and their heights are 4 and $3 \mathrm{~mm}$, respectively. Parts B and C are each $10 \mathrm{~mm}$ long and their diameters are 1 and $1.5 \mathrm{~mm}$, respectively. The outer diameter of the brass part D is $3 \mathrm{~mm}$ to conform with the inner diameter of part E. The diameter of the two holes in both parts $\mathrm{D}$ and $\mathrm{F}$ (diameter $2.5 \mathrm{~mm}$ ) corresponds with the diameter of the two stainless steel parts B. Additionally, a borosilicate glass tube (part $\mathrm{H}$ ) is put on top of the mold base plate (part A) to form the circumference of the PDMS casing. The tube has an inner diameter of $15 \mathrm{~mm}$.

For the molding of the PDMS casing, Sylgard 184 elastomer and curing agent (10:1 wt.\%) are mixed and poured onto the mold configuration as shown in Fig. 1(b). The PDMS mixture is degassed and cured at $80^{\circ} \mathrm{C}$ for $30 \mathrm{~min}$ in a convection oven. Afterward, the PDMS casing is removed from the mold, cleaned with $70 \%$ ethanol (Sigma), and thoroughly rinsed with ultrapure water (Millipore). In the second step of the molding process, the PDMS casing is placed onto the mold configuration shown in Fig. 1(c) to form the hydrogel barrier. Agarose (Sigma) is used for the barrier material. Agarose powder is dissolved in phosphate buffered saline (PBS) (Sigma) at $2 \%$ and heated to $80^{\circ} \mathrm{C}$. The barrier is formed by inserting the agarose in the PMDS casing at the inlet resulting from part $\mathrm{C}$ [indicated by the arrow in Fig. 2(a)]. After gelation of the agarose, the PDMS with the integrated hydrogel barrier is removed from the mold. The resulting bioreactor is adhered to a glass or MEA surface (Fig. 3).

\section{B. Transport through the barrier}

The bioreactor is fabricated as described above and adhered to a microscope glass slide $\left(76.2 \times 25.4 \times 1 \mathrm{~mm}^{3}\right)$. The channel and culture chamber are filled with PBS to avoid that the hydrogel dries out prior to the measurement. Before use, the PBS is removed strictly from the channel. A $(1 \mathrm{mg} / \mathrm{ml})$ fluorescein isothiocyanate conjugated bovine serum albumin (FITC-BSA) solution in PBS is injected into the channel. Diffusion of the labeled protein into the agarose
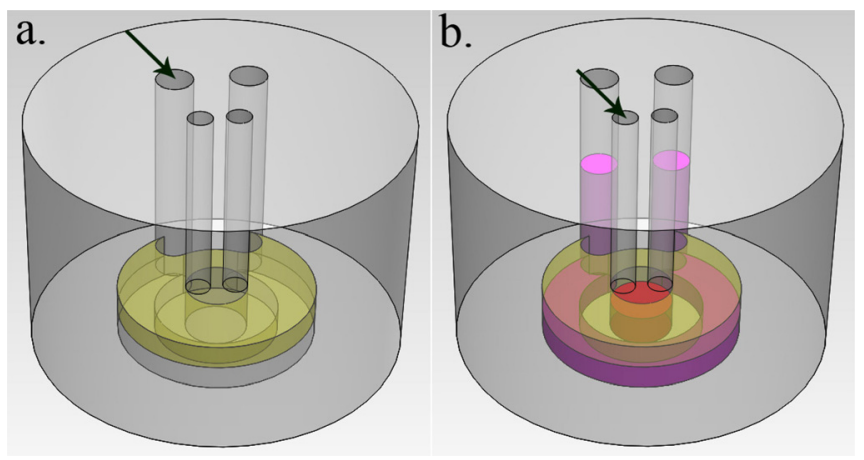

FIG. 2. (Color online) Schematics of the hybrid bioreactor. The arrow indicates the position of the inlets during fabrication and use. (a) The bioreactor after the integration of the hydrogel barrier. (b) The bioreactor with the inserted Matrigel cell culture (in the center) and culture medium in the surrounding channel. 


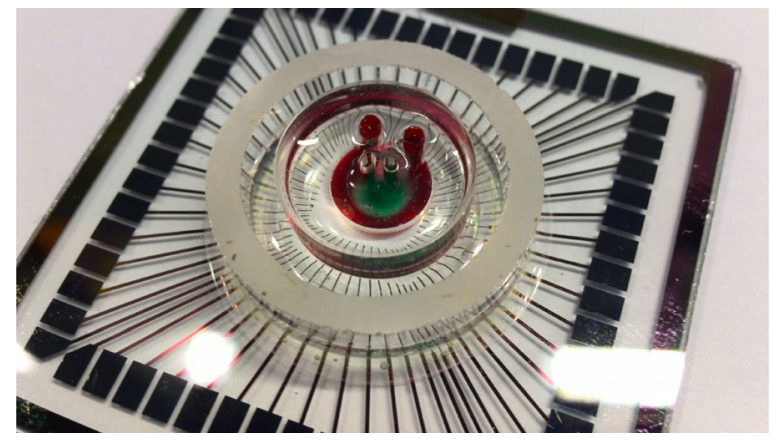

FIG. 3. (Color online) Bioreactor can be adhered to the commercially available MEA simply by the adhesive properties of the PDMS casing, resulting in a leak-tight connection between the PDMS casing and the MEA, while the culture chamber is aligned to the electrode array. Colored dye solutions are injected into the bioreactor to indicate the channel and the culture chamber.

barrier is observed by a fluorescence microscope (Leica DM IL LED) over distance at $25^{\circ} \mathrm{C}$. The fluorescent images for quantifying the diffusion through the barrier were analyzed using IMAGEJ software for intensity profiling at three different locations in the bioreactor. The obtained data were fitted to a Gaussian function for dimensional diffusion with ORIGIN 7.5 software to simulate the diffusion profiles for further analyzes. $^{18}$

\section{Cell culture in the bioreactor}

\section{Static bioreactor culture}

The bioreactor is adhered to a microscope glass slide and PBS is injected into the ring shaped channel [indicated by the arrow in Fig. 2(a)]. Cortical cells (taken from the entire cortex) were obtained from newborn Wistar rats. The cells are mixed with reduced growth factor Matrigel (DB biosciences) to 4.5 $\times 10^{6}$ cells $/ \mathrm{ml}$ and kept on ice. A volume of $5 \mu \mathrm{l}$ cells in Matrigel is injected into the culture chamber through the inlet resulting from part B [indicated by the arrow in Fig. 2(b)]. Subsequently, the bioreactor is placed in an incubator $\left(37^{\circ} \mathrm{C}\right.$, $5 \% \mathrm{CO}_{2}$ ) for $1 \mathrm{~h}$ to initiate gelation. Afterward, the PBS is replaced by chemically defined $\mathrm{R} 12$ culture media. ${ }^{19}$ The bioreactor is placed in an incubator $\left(37^{\circ} \mathrm{C}, 5 \% \mathrm{CO}_{2}\right)$ and the medium is refreshed every other day.

\section{Static control culture}

As a control, cell cultures with a cell density conform the bioreactor are inserted into inlets $(2.5 \mathrm{~mm}$ diameter $)$ in a $2 \mathrm{~mm}$ thick layer of agarose hydrogel, which is deposited on glass slides. Chemically defined R12 culture media is added on top of the culture and the surrounding agarose. The control culture is placed in an incubator $\left(37^{\circ} \mathrm{C}, 5 \% \mathrm{CO}_{2}\right)$ and the medium is refreshed every other day.

\section{Viability staining}

After 6 days of culturing, both bioreactor and control are stained for viability (Sigma, Cellstain double staining kit) to study the survival of cells in the bioreactor. The samples are washed three times with PBS, after removal of the culture medium. Subsequently, a solution of $1 \mu \mathrm{M}$ calcein-AM and $2 \mu \mathrm{M}$ ethidium homodimer is inserted into the channel [indicated by the arrow in Fig. 2(a)] or in case of the control on top of the culture. Both cultures are incubated for $30 \mathrm{~min}$, followed by repetition of the washing steps. Viable and dead cells are observed by a fluorescence microscope (Leica DM IL LED) and counted for the peripheral and the center regions of the culture.

\section{EXPERIMENTAL RESULTS}

\section{A. Bioreactor fabrication}

The fabrication of the hydrogel/PDMS hybrid bioreactor has been successfully realized by a two-step molding process. The principle of the two-step molding process is based on utilizing two different mold configurations that are aligned to each other by interchangeable parts in a base plate. First, a casing of PDMS is molded using the parts depicted in Fig. 1(b). Subsequently, the mold configuration shown in Fig. 1(c) is used together with the PDMS casing to form the hydrogel barrier from agarose. This specific shape of the barrier (see schematic of the bioreactor in Fig. 2) ensures a fixed position inside of the PDMS casing. The mold material is chosen because of its favorable mechanical and chemical resistance and temperature coefficient. The assembly of the mold from interchangeable parts (rather than machining a single component mold) enables a smooth surface finish for the mold base plate part A [Fig. 1(a)] and simple alignment for the second molding step forming the barrier inside of the casing. This surface finish guarantees a leak-tight connection, when the add-on bioreactor is adhered to the glass or MEA surface (Fig. 3). Here, colored dye solutions are injected into the bioreactor to show the different zones, respectively, the channel, the barrier, and the culture chamber.

\section{B. Characterization of the barrier}

The barrier is integrated to separate the fluidic channel from the cell culture while maintaining mass transport into the culture. To test this hypothesis, we used agarose hydrogel, which is a commercially available sol-gel, relative inexpensive, and biocompatible. Further, it has been shown elsewhere that it prevents adhesion of cells and migration out of the culture at a mass volume ratio of $2 \% .{ }^{20}$ Within a static culture setup, mass transport through the barrier is observed by diffusion of FITC-BSA (Fig. 4). The simulated diffusion profiles are found to be in agreement with the experimental results $(\mathrm{R}=0.97 \pm 0.02)$. The profiles show a decrease in FITC-BSA initial concentration at the channel side and an increase in FITC-BSA concentration at the interface of the culture chamber, as a result of the static setup. This result shows a diffusion coefficient of an average value of $2.2 \times 10^{-7} \mathrm{~cm}^{2} \mathrm{~s}^{-1}$, which is in agreement with values reported elsewhere. ${ }^{21}$

\section{Cell culture in the bioreactor}

The add-on bioreactor was used to culture neuronal cells in Matrigel. To validate the function of the barrier within the 

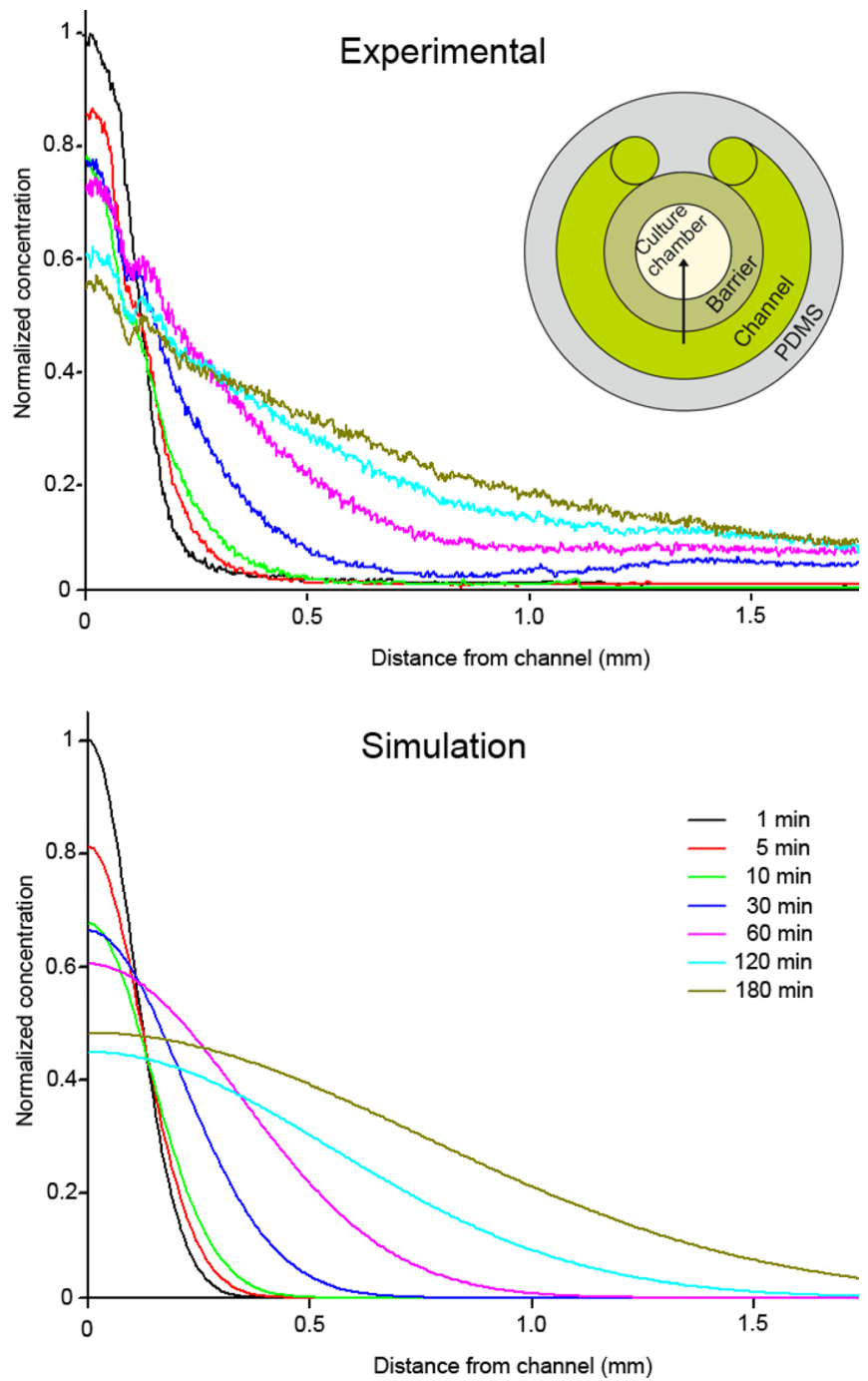

FIG. 4. (Color online) Profiles of the diffusion of FITC-BSA $(1 \mathrm{mg} / \mathrm{ml}$, $66 \mathrm{kDa}$ ) for the experimental and simulated data. The data are collected from the microscope intensity images according to the pathway following the channel toward the culture chamber, thereby crossing the agarose barrier (this pathway is indicated by the arrow in the schematic insert). The finite FITC-BSA concentration in the channel results in a decrease of initial concentration as the fluorescent protein diffuses from the channel toward the barrier and culture chamber.

bioreactor for 3D cell culturing, we compared the setup with a specifically designed control experiment. This experiment allowed us to evaluate cell viability dependent on the degree of supply of culture medium. The diffusion of culture media in the bioreactor is limited by the barrier. However, in the control culture medium is added directly on top of the culture.

Cells in both the bioreactor and the control were distributed in a 3D fashion within the Matrigel. For the bioreactor, an increased cell density is observed in the peripheral region compared to the center of the culture (Fig. 5). We counted viable and dead cells for the total population, peripheral and center regions (Fig. 6). Even with the integrated barrier in the bioreactor, an enhanced cell viability is observed compared to the control. For the bioreactor, the fraction of viable cells in the peripheral region is higher compared to the

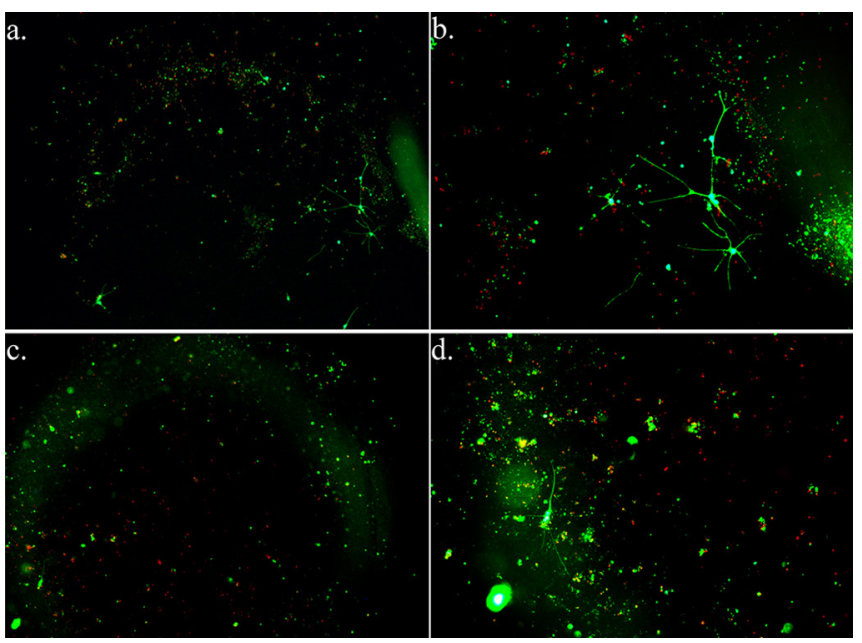

FIG. 5. (Color online) Cells cultured in Matrigel in the bioreactor [(a) and (b), respectively, 10 and $20 \times$ magnification], a higher amount of cells is seen in the peripheral region compared to the center. The control [(c) and (d), respectively, 10 and $20 \times$ magnification] shows a comparable amount of cells in both regions. Cells in the bioreactor and the control were cultured for 6 days and afterward stained by Calcein AM for viable cells (green) and ethidium homodimer for dead cells (red).

center. For the control culture, these fractions are of the same order of magnitude in both regions.

\section{DISCUSSION}

This work has demonstrated the fabrication of a bioreactor, which can be used to facilitate 3D culturing on a commercially available MEA. The bioreactor can be reversibly adhered to a glass or MEA surface before insertion of the cell culture material. This specific design allows direct gelation of the 3D cell culture on top of the microelectrodes of a MEA. Thanks to the integrated hydrogel barrier in the bioreactor, mass transport into a $3 \mathrm{D}$ culture chamber can be realized without applying shear forces to the cells. The hybrid bioreactor design has the potential for batch processing by means of the two-step molding process, which could

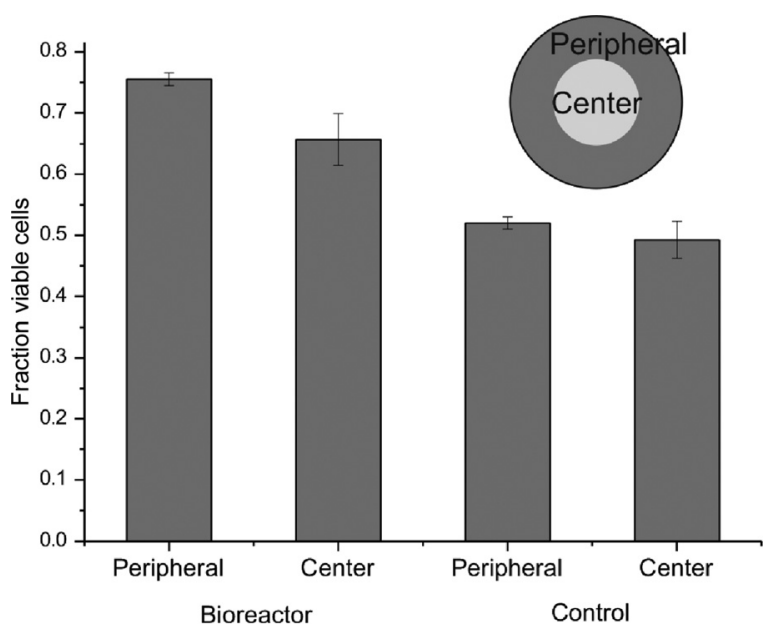

FIG. 6. Graphical representation of viable cell fractions of the peripheral and center regions of both bioreactor and control. Cells were cultured (6 days) in Matrigel in the bioreactor and control (both performed in triplicate). 
be used to enable high throughput screening. To accommodate specific biological experiments, the barrier material in the bioreactor can easily be varied. For example, different types or concentrations of hydrogel can be used to alter mass transport or to add functional properties. The observation of diffusion of FITC-BSA in the agarose hydrogel provided us with an initial indication of culture medium inflow (Fig. 4). The size of FITC-BSA (66 kDa) is significantly larger than most nutrient molecules in the culture medium. Also, the stokes-radius of the BSA is around $3.5 \mathrm{~nm}$, while the pore size in the $2 \%$ agarose is around $80 \mathrm{~nm} .{ }^{21}$ Therefore, diffusion for these nutrients is expected not to be restricted by the barrier and to have higher diffusion rates than FITC-BSA, rendering our design fit for 3D cell culture. Nevertheless, culturing is a process at a much larger time scale than the diffusional process (rather weeks than minutes) with regular intervals for medium refreshment. Therefore, the time required toward a diffusion equilibrium in the bioreactor is ought to be shorter in comparison to a total nutrient depletion in the culture chamber. In other words, starving of cells in the culture chamber is not likely due to the integration of a hydrogel barrier.

Subsequently, we validated the bioreactor for cell viability by culturing rat cortical cells. Figure 5 shows the distribution of cells for the bioreactor and the control. Here, we observed a higher fraction of viable cells in the peripheral region for the bioreactor compared to the control. The fraction of viable cells for both regions in de bioreactor is higher than in the control, which might be due to differences in the cell seeding conditions. The difference in viability between the regions in the bioreactor is an expected result, as the mass transport of culture medium in the control is evenly distributed compared to the bioreactor. These results indicate that diffusion alone in the static culture in the bioreactor is not sufficient for the delivery of nutrients to acquire a homogenous distribution in the culture chamber. To obtain a more homogenous distribution, the barrier thickness can be reduced to decrease the residence time of a nutrient molecule in the barrier. Also, by a continuous and infinite flow of culture medium, the concentration of nutrients in the bioreactor could reach an increased homogenous distribution of nutrients in all zones, compared to the static setup which we tested in this paper.

\section{SUMMARY AND CONCLUSIONS}

A rapid, reproducible, and inexpensive fabrication process of a microfluidic hydrogel/PDMS hybrid bioreactor has been developed, which enables 3D culturing of cells atop of a commercially available MEA. The fabrication of the
PDMS casing permits the leakage free, reversible adhesion of the bioreactor to the surface of a MEA. The two-step molding procedure enables the successful integration of a hydrogel barrier, preventing shear stress while sustaining transport of nutrients.

FITC-BSA is used as a model for nutrient supply through the barrier molded from agarose. This model shows a diffusion into the culture chamber without leakage of the barrier. Furthermore, the bioreactor enabled static 3D cell culturing of rat cortical cells in Matrigel on a glass surface with a cell viability well above $60 \%$.

\section{ACKNOWLEDGMENTS}

The authors gratefully acknowledge financial support by the European Research Council Grant MESOTAS No. 280281. They also like to thank Gerco Hassink from the Biomedical Signals and Systems group of the University of Twente for the isolation of the brain cells.

${ }^{1}$ D. D. Allen, R. Caviedes, A. M. Cardenas, T. Shimahara, J. SeguraAguilar, and P. A. Caviedes, Drug Dev. Ind. Pharm. 31, 757 (2005).

${ }^{2}$ V. Vickerman, J. Blundo, S. Chung, and R. Kamm, Lab Chip 8, 1468 (2008).

${ }^{3}$ A. Abbott, Nature 424, 870 (2003).

${ }^{4}$ F. Pampaloni, E. G. Reynaud, and E. H. K. Stelzer, Nature Rev. Mol. Cell Biol. 8, 839 (2007).

${ }^{5}$ G. Mazzoleni, D. Di Lorenzo, and N. Steimberg, Genes Nutr. 4, 13 (2009).

${ }^{6}$ M. W. Tibbitt and K. S. Anseth, Biotechnol. Bioeng. 103, 655 (2009).

${ }^{7}$ J. Khandurina and A. Guttman, Curr. Opin. Chem. Biol. 6, 359 (2002).

${ }^{8}$ R. Gomez-Sjoberg, A. A. Leyrat, D. M. Pirone, C. S. Chen, and S. R. Quake, Anal. Chem. 79, 8557 (2007).

${ }^{9}$ M. H. Wu, S. B. Huang, and G. B. Lee, Lab. Chip 10, 939 (2010).

${ }^{10}$ G. W. Gross, E. Rieske, G. W. Kreutzberg, and A. Meyer, Neurosci. Lett. 6, 101 (1977).

${ }^{11}$ S. M. Potter and T. B. DeMarse, J. Neurosci. Methods 110, 17 (2001).

${ }^{12}$ M. D. Eggers, D. K. Astolfi, S. Liu, H. E. Zeuli, S. S. Doeleman, R. McKay, T. S. Khuon, and D. J. Ehrlich, J. Vac. Sci. Technol. B 8, 1392 (1990).

${ }^{13}$ E. Sofla, E. Seker, J. P. Landers, and M. R. Begley, J. Appl. Mech. 77, 031007 (2010).

${ }^{14}$ N. Bhattacharjee, N. Li, T. M. Keenan, and A. Folch, Integr. Biol. 2, 669 (2010).

${ }^{15}$ Y. Shin, S. Han, J. S. Jeon, K. Yamamoto, I. K. Zervantonakis, R. Sudo, R. D. Kamm, and S. Chung, Nat. Protoc. 7, 1247 (2012).

${ }^{16}$ B. Mosadegh et al., Langmuir 23, 10910 (2007).

${ }^{17}$ R. Luttge, E. J. W. Berenschot, M. J. de Boer, D. M. Altpeter, E. X. Vrouwe, A. van den Berg, and M. Elwenspoek, J. Microelectromech. Syst. 16, 872 (2007).

${ }^{18}$ Q. H. Wei, C. Bechinger, and P. Leiderer, Science 287, 625 (2000).

${ }^{19}$ H. J. Romijn, F. van Huizen, and P. S. Wolters, Neurosci. Biobehav. Rev. 8, 301 (1984).

${ }^{20}$ A. P. Balgude, X. Yu, A. Szymanski, and R. V. Bellamkonda, Biomaterials 22, 1077 (2001).

${ }^{21}$ A. Pluen, P. A. Netti, R. K. Jain, and D. A. Berk, Biophys. J. 77, 542 (1999). 